THE JOURNAL OF SYMBOLIC LOGIC

Volume 00, Number 0, XXX 0000

\title{
DETERMINACY IN STRONG CARDINAL MODELS
}

\author{
P. D. WELCH
}

\begin{abstract}
We give limits defined in terms of abstract pointclasses of the amount of determinacy available in certain canonical inner models involving strong cardinals. We show for example: Theorem A $\operatorname{Det}\left(\Pi_{1}^{1}-\mathrm{IND}\right) \Rightarrow$ there exists an inner model with a strong cardinal. Theorem $\mathrm{B} \operatorname{Det}(\mathbf{A Q I}) \Rightarrow$ there exist type-1 mice and hence inner models with proper classes of strong cardinals where $\Pi_{1}^{1}$-IND (AQI) is the pointclass of boldface $\Pi_{1}^{1}$-inductive (respectively arithmetically quasi-inductive) sets of reals.
\end{abstract}

$\S 1$. Introduction and Preliminaries. It is well known that determinacy of GaleStewart games ( $c f[7]$ ) beyond the Borel hierarchy requires strong axioms of infinity, that are generally stated in terms of large cardinals, or embedding properties of the universe $V$ of all sets of mathematical discourse. In the presence of such axioms canonical constructions of inner models result in such models having a spectrum of properties, depending on the strength of the axioms assumed. In general the stronger the axioms the more involved the structure of the models can be become. One measure of that complexity is that of the definability of the wellorder of the real continuum in the model. In Gödel's $L$ this wellordering is $\Delta_{2}^{1}$ ([7] ). More complicated models require a more complicated ordering. As is well known the full Axiom of Determinacy, $A D$, contradicts the Axiom of Choice. However this works level by level: Choice at a certain level of definability rules out the determinacy of games at roughly that level too. Hence, given an inner model $M$, determining the complexity of the wellordering in that model (which here is related to the complexity of the levels of the model - we work assuming that certain strong axioms do not hold) gives us via Kechris's Theorem 2 below, an upper bound as to how much determinacy to expect to be available in that model. To state our results in a contrapositive form here: if such and such amount of determinacy holds in a canonical inner model then that model must have a wellordering sufficiently complex to ensure that those strong axioms must hold in an inner model.

This note should be viewed as revisiting a paper of Steel [9], where he outlined all of the above discussion and thereby sought to ascertain limits on the amount of determinacy holding in the Mitchell style core models of that era, which contained coherent

Received by the editors November 10th, 2009.

The author would like to express his thanks to the EPSRC for their INTERACT grant GR/S72399. and would also like to warmly thank the Academy of Sciences, Beijing for their hospitality in March 2004 when this paper was (mostly) written

(c) 0000, Association for Symbolic Logic 0022-4812/00/0000-0000/\$00.00 
sequences of normal measures. They were thus of the form $L[\overrightarrow{\mathcal{U}}]$ where $\overrightarrow{\mathcal{U}}$ was such a measure sequence. Thus (to quote just one of his results) we have:

THEOREM 1. (Steel) [9] If $\overrightarrow{\mathcal{U}}$ is a sequence of coherent measures then in $L[\overrightarrow{\mathcal{U}}] \Gamma_{0^{-}}$ Determinacy fails.

We have to specify the pointclass $\Gamma_{0}$ for the theorem to be meaningful. The pointclasses are meant to be strictly within $\Delta_{2}^{1}$, as it was well known that $\Delta_{2}^{1}$-Determinacy fails in such $L[\overrightarrow{\mathcal{U}}]$-models. The nature of the pointclass is influenced by the means of proof: he uses a Friedman style game of comparing two premice which disagree about the ordering of their reals, inside a certain admissible set with certain reflection properties: the stronger the premice, the stronger the reflection properties of the admissible set needed to realise their comparison. Thus for Theorem 1 he defines:

DEFINITION 1. Let $\Gamma_{0}$ be the following pointclass of sets of reals: $A \subseteq \mathbb{R}$ is in $\Gamma_{0}$ if and only if there is a $\Sigma_{1}$ formula $\varphi\left(v_{0}\right)$ so that

$$
\forall x\left(x \in A \longleftrightarrow L_{\alpha(x)}[x] \models \varphi[x]\right)
$$

where $\alpha(x)$ denotes the next $x$-admissible ordinal greater than the least $\Pi_{4}(x)$-reflecting ordinal.

The choice of $\alpha$ here is because he proves that the comparison of two countable premice $M, N$ must terminate before the least ordinal which is $\Pi_{4}$-reflecting in (a real code for) $M, N$ - this termination may well be because one of them is seen to be illfounded. As he puts more conditions on $\overrightarrow{\mathcal{U}}$, so he can weaken the amount of reflection, and so smaller ordinals than $\alpha$ can be chosen. He is thus implicitly proving results about how much set theory is needed to prove comparison of certain classes of mice. We shall need to consider reflection beyond models of KP: we thus denote by $\Sigma_{2}-\mathrm{KP}$, the usual Kripke-Platek axioms which are augmented to allow for instances of $\Delta_{2}$-Separation, and $\Sigma_{2}$-Collection - and similarly for $\Sigma_{3}$-KP. Underlying the proofs is the following theorem:

THEOREM 2. (Kechris)[6] If there is a $\Gamma$-wellorder of $\mathbb{R}$ then $\Gamma$-Determinacy fails.

We use the following definition to delineate our pointclasses.

DEFINITION 2. For $i \geq 1$ let $\Gamma_{i}$, be the following pointclasses of sets of reals:

$A \subseteq \mathbb{R}$ is in $\Gamma_{i}$, if and only if there is a $\Sigma_{1}$ formula $\varphi\left(v_{0}\right)$ so that

$$
\forall x\left(x \in A \longleftrightarrow L_{\Sigma(i, x)}[x] \models \varphi[x]\right)
$$

where $\Sigma(i, x)$ denotes the least ordinal $\Sigma$ either $x$-admissible, or a limit of $x$-admissibles, so that $L_{\Sigma}[x]$ has a proper $\Sigma_{i}$ substructure $L_{\zeta}[x] \prec_{\Sigma_{i}} L_{\Sigma(i, x)}[x]$. (In each case we set $\zeta(i, x)$ to be the ordinal height of this substructure.)

Note: $\Sigma(1, x)$ is in fact (a successor) $x$-admissible; $\Sigma(2, x)$ is always a limit of $x$ admissibles, but is not itself $x$-admissible (Cor. 3.4 of [12]). For $i>2 \Sigma(i, x)$ is an $x$ admissible, that is a limit of such (just note that the axioms of $K P$ are $\Pi_{3}$-formalisable, hence $\zeta(i, x)$ 's being an admissible limit of admissibles goes up to $\Sigma(i, x)$ ).

We shall compare the first two of these pointclasses at least with pointclasses defined via operators. Suppose we have a map $\Gamma: \mathcal{P}(\mathbb{N}) \rightarrow \mathcal{P}(\mathbb{N})$. We say this is recursive, 
arithmetic, $\Sigma_{1}^{1}$ etc. if the relation ' $n \in \Gamma(X)$ ' is definable in that manner. We may define iterates:

$$
\Gamma^{0}(z)=z ; \Gamma^{\alpha+1}(z)=\Gamma\left(\Gamma^{\alpha}(z)\right) ; \Gamma^{\lambda}(z)=\bigcup_{\alpha<\lambda} \bigcap_{\alpha<\beta<\lambda} \Gamma^{\beta}(z)(\lim (\lambda) \vee \lambda=\infty)
$$

If $\Gamma$ is monotone (that is $A \subseteq B \rightarrow \Gamma(A) \subseteq \Gamma(B)$ ) or simply progressive (that is $A \subseteq \Gamma(A)$ ) one reaches a fixed point $\Gamma^{\infty}(z)$, because in this case we have $\alpha<\beta \rightarrow$ $\Gamma^{\alpha}(z) \subseteq \Gamma^{\beta}(z)$ (the limit stage being a simple union). Otherwise it can easily be seen to have a countable stability point $\zeta$ so that $\Gamma^{\zeta}(z)=\Gamma^{\infty}(z)$ (and indeed a proper class of such with a fixed periodicity). We identify $\mathcal{P}(\mathbb{N})$ with $\mathbb{R}$.

Definition 3. A set $A \subseteq \mathbb{R}$ is $\Pi_{1}^{1}$-IND iff there exists a $\Pi_{1}^{1}$ function $\Gamma: \mathbb{R} \times \mathbb{R} \rightarrow$ $\mathbb{R}$, so that for any $x$ setting $\Gamma_{x}(y)={ }_{d f} \Gamma(x, y)$ ) defines an inductive operator (not necessarily monotone) in $x$ for which:

$$
\forall x \in \mathbb{R}\left(x \in A \longleftrightarrow 0 \in \Gamma_{x}^{\infty}(\varnothing)\right) .
$$

DEFINITION 4. A set $A \subseteq \mathbb{R}$ is $\mathrm{AQI}$ iff there is some arithmetic operator $\Gamma: \mathbb{R} \rightarrow \mathbb{R}$, so that:

$$
\forall x \in \mathbb{R}\left(x \in A \longleftrightarrow 0 \in \Gamma^{\infty}(x)\right) .
$$

Using [1] Theorems 1.19 and 10.7, one may show that a set $a \subseteq \mathbb{N}$ is $\Pi_{1}^{1}$-inductive (that is, it is (1-1) reducible to a fixed point of an inductive $\Pi_{1}^{1}$-operator) if it is $\Sigma_{1}$ definable over $L_{\Sigma(1, \varnothing)}$. The higher type definition above yields (generalising those arguments) that $\Gamma_{1}=\Pi_{1}^{1}$-IND. Moving up a level, Burgess coined the phrase 'arithmetically quasi-inductive,' [2], and he proved (his Theorem 14.1) a set $a \subseteq \mathbf{N}$ to be AQI iff $a \in \Sigma_{2}\left(L_{\Sigma(2, \varnothing)}\right)$. The higher type definition of AQI just given widens this definition to $\mathbb{R}$, and the generalisation of his result is that $\mathrm{AQI}=\Gamma_{2}$.

If we define a pointclass by AQI operators reaching fixed points, i.e. where $\Gamma^{\alpha}(x)=$ $\Gamma^{\alpha+1}(x)$ then this defines a slightly smaller class: reaching a fixed point is a $\Sigma_{1}$ fact, (when considered over $L_{\Sigma(2, x)}[x]$ ) and thus the $\Sigma_{2} \varphi$ in (1) can be replaced by a $\Sigma_{1}$ formula. We should point out that all the classes mentioned above are Spector pointclasses in the sense of Moschovakis [7].

The proof of the Theorem 6 will show that the canonical wellordering on mice is arithmetically quasi-inductive when restricted to a certain class of mice - the "type- 0 mice." (Indeed it is in the subclass of AQI sets defined by operators reaching fixed points.) This represents a partial answer to a question raised in [13] as to how complex the notion of ITTM higher type recursion can be, as the latter is an example of a arithmetical quasi-induction which is complete for that pointclass.

Teasing these definitions apart we shall have that there is an arithmetic (indeed recursive) $\Gamma$ so that if $z$ codes a pair of countable type- 0 premice $\langle M, N\rangle$ then on comparison either $M \unlhd N$, or $M$ becomes illfounded before $N$ does iff $0 \in \Gamma^{\infty}(z)$.

This brings us to defining the classes of premice we shall be considering. Apart from the first which predates the others, these definitions come from [3].

DEFINITION 5. Let $M$ be a premouse. We say $M$ is a non-overlapping premouse, if for every $\kappa<\mathrm{On}^{M}$, if $\kappa$ is the critical point of some extender on $E^{M}$, then $\{\tau<$ $\left.\kappa \mid o^{M}(\tau) \geq \kappa\right\}=\varnothing$. (Here $o^{M}(\tau)={ }_{d f}\left\{\lambda \mid\right.$ there exists an extender $E$ on the $E^{M}$ sequence with $\operatorname{crit}(E)=\tau$ and $\operatorname{lh}(E)=E(\operatorname{crit}(E))=\lambda\}$.) 
The least mouse that is overlapping, if it exists, is denoted 0 . By iterating the measure of 0 that is so overlapped through all the ordinals, one generates an inner model with a single strong cardinal, together with a closed and unbounded class of indiscernibles for it.

DEFINITION 6. Let $M$ be a premouse. We say $M$ is a type-0 premouse, if for every $\kappa<\mathrm{On}^{M}$, if $\kappa$ is the critical point of some extender on $E^{M}$, then $\kappa$ is of type- 0 in $M$, where we define:

$\kappa$ is of type- 0 in $M \Longleftrightarrow$

$\exists \nu \leq \operatorname{On}^{M}\left(\kappa=\operatorname{crit}\left(E_{\omega \nu}^{M}\right) \wedge\left\{\tau<\kappa \mid o^{M}(\tau) \geq \kappa\right\}\right.$ is bounded in $\left.\kappa\right)$.

A type-0 mouse can thus generate an inner model with at most set-many strong cardinals. Feng and Jensen identify a stronger type:

DEFINITION 7. Let $M$ be a premouse. We say $M$ is a type-1 premouse, if for every $\kappa<\mathrm{On}^{M}$, if $\kappa$ is the critical point of some extender on $E^{M}$, then $\kappa$ is of type-0 or type-1 in $M$, where we define:

$\kappa$ is of type $\geq 1$ in $M \Longleftrightarrow$

$\exists \nu \leq \mathrm{On}^{M}\left(\kappa=\operatorname{crit}\left(E_{\omega \nu}^{M}\right) \wedge\left\{\tau<\kappa \mid o^{M}(\tau) \geq \kappa \wedge \tau\right.\right.$ is of type-O $\}$ is unbounded in $\kappa)$.

$\kappa$ is of type $\geq 2$ in $M \Longleftrightarrow$

$\exists \nu \leq \mathrm{On}^{M}\left(\kappa=\operatorname{crit}\left(E_{\omega \nu}^{M}\right) \wedge\left\{\tau<\kappa \mid o^{M}(\tau) \geq \kappa \wedge \tau\right.\right.$ is of type $\left.\geq 1\right\}$ is unbounded in $\kappa)$.

$\kappa$ is of type- 1 in $M \Longleftrightarrow \kappa$ is of type $\geq 1$ but not of type $\geq 2$.

We shall use the following facts about finding branches through normal iterations trees for type- 0 and type- 1 mice. For type- 0 , if $M$ is such a mouse and

$$
\left\langle\left\langle M_{i}\right\rangle_{i<\theta},\left\langle\nu_{i}\right\rangle_{i<\theta},\left\langle\eta_{i}\right\rangle_{i+1<\theta},\left\langle\pi_{i, j}^{M}\right\rangle_{i \leq j<\theta}, T\right\rangle
$$

is a normal iteration of $M=M_{0}$ with underlying tree order $<_{T}$ on $\theta$ given by $T$, the 'unique $<_{T}$-predecessor' function, then the iteration is "almost linear": to find the models on the unique cofinal branch, $b$ say, one only has to put $i \in b$ if $i$ is maximal amongst those possibly finitely many $i^{\prime}$ which share the same $T$-predecessor with $i$ : $i \in b \Longleftrightarrow i=\max \left\{i^{\prime} \mid T\left(i^{\prime}\right)=T(i)\right\}$ (where $T(j)$ is defined as the immediate $T$-predecessor of $j)$. To put it another way $i \in b \Longleftrightarrow \forall j>i\left(i<_{T} j\right)$. This we can formally express as follows:

THEOREM 3. Let $M$ be a type-O premouse, and suppose that $\mathcal{T}$ is a normal iteration tree on $M$ of limit length $\theta$. Then $\mathcal{T}$ has a unique cofinal branch $b=b_{\mathcal{T}}=\{i<\theta \mid$ $\left.\forall k<\theta\left(k>i \longrightarrow i<_{T} k\right)\right\}$.

For mice of type-1 the branching possibilities are slightly more complicated but Feng and Jensen have:

THEOREM 4. ([3] Thm. 2.4) Let $M$ be a type-1 premouse, and suppose that $\mathcal{T}$ is a normal iteration tree on $M$ of limit length $\theta$. Then $\mathcal{T}$ has a unique cofinal branch $b=b_{\mathcal{T}}=\left\{i<\theta \mid \forall k<\theta \exists j>k\left(i<_{T} j\right)\right\}$.

In an iteration tree based on type- 0 premice, each point of the tree can have at most finitely many immediate successors. Such iterations are almost linear. Type- 1 premice can have iteration trees that are infinitely branching from a single point ( $c f[3]$ Ex.2.1.) 
We prove here the following on $L[\vec{E}]$ models which admit strong cardinals, and strong limits of such (but not much more).

Theorem 5. Suppose $V=L[\vec{E}]$ and 0 does not exist. Then $\Gamma_{1}$-Determinacy fails.

TheOREM 6. Suppose $V=L[\vec{E}]$ and there are only type-0 mice. Then $\Gamma_{2}$-Determinacy fails.

THEOREM 7. Suppose $V=L[\vec{E}]$ and there are only mice of type $\leq 1$. Then $\Gamma_{3}$ Determinacy fails.

In the first of these theorems we recall that all comparison iterations are truly linear, and iteration trees are not needed. There are thus no problems in determining direct limit models in limit length trees. For the latter two theorems the mice involved are still very weak, and so defining cofinal branches can be, and ultimately is, still easy.

\section{$\S 2$. The proofs.}

Lemma 1. Let $\mathfrak{A} \models \mathrm{KP}$, with $\mathfrak{A}$ transitive, and let $M, N \in \mathfrak{A}$ be type-0 premice. Let $\theta=\mathrm{On}^{\mathfrak{A}}$. Suppose $M, N$ are coiterable for $\mu \leq \theta$ many steps (meaning that the models are wellfounded on both sides for this length of coiteration). Then the iteration trees arising in the comparison

$$
\mathcal{T}=\left\langle\left\langle M_{i}\right\rangle_{i<\mu},\left\langle\nu_{i}\right\rangle_{i<\mu},\left\langle\eta_{i}\right\rangle_{i+1<\mu},\left\langle\pi_{i, j}^{M}\right\rangle_{i \leq j<\mu}, T\right\rangle
$$

and

$$
\mathcal{U}=\left\langle\left\langle N_{i}\right\rangle_{i<\mu},\left\langle\nu_{i}\right\rangle_{i<\mu},\left\langle\eta_{i}\right\rangle_{i+1<\mu},\left\langle\pi_{i, j}^{N}\right\rangle_{i \leq j<\mu}, U\right\rangle,
$$

are definable by a $\Delta_{1}^{\mathfrak{A}}(\{M, N\})$-definable recursion, with the final branches $b, c \subseteq \theta$ being $\Pi_{1}^{\mathfrak{A}}(\{M, N\})$-definable.

Proof: We first make the remark that if $\mathcal{T}\lceil k+1, \mathcal{U}\lceil k+1$ have been defined inside of $\mathfrak{A}$ the choice of which extender(s) to use at the next stage is entirely effective from a KP-point of view: it is a $\Delta_{1}$ process to look for the index $\nu_{k}$ of least difference in $E^{M_{k}}, E^{N_{k}}$, and apply the extender to the relevant model to form its ultrapower.

At a limit stage, given the branch along which to form a direct limit, if the direct limit is wellfounded, then its transitive isomorph is an element of $\mathfrak{A}$; it is then a $\Delta_{1}$ query to check whether a given candidate transitive set $M_{\lambda}$ or $N_{\lambda}$ is isomorphic to that direct limit. However defining the correct relevant branch(es) is also a $\Delta_{1}$ enquiry of the set which is the tree ordering $T \nmid \lambda$ defined to date. Theorem 3 (and the remark just before it) shows that we can define these branches in a $\Pi_{1}$-manner over $\mathfrak{A}$ if $\mu=\theta$. Q.E.D.

Lemma 2. Let $\mathfrak{A} \models \mathrm{KP}, M, N \in \mathfrak{A}, \theta$ be as above, excepting that $M, N$ may be type-1 premice. Then the iteration trees arising in the comparison are definable by a $\Delta_{1}^{\mathfrak{A}}(\{M, N\})$-recursion, with $b, c \subseteq \theta$ the cofinal branches being $\Pi_{2}^{\mathfrak{A}}(\{M, N\})$.

Proof: The only difference here is that additional complexity of the mice requires a more sophisticated definition of cofinal branch. Here we have, for limit $\mu$ (see [3] Thm 2.4):

$i \in b \Longleftrightarrow \forall k<\mu \exists l<\mu\left(k \leq l \wedge i<_{T} l\right)$

(and similarly for $c, \mathcal{U}$ - we should perhaps remark that this is nothing to do with the comparison process, this is just the only way to define the cofinal branch in a normal 
iteration tree of a type-1 mouse $M$ - as [3] Thm 2.4 proves.) The displayed equivalence gives our result.

Q.E.D.

We now see how much set theory will be required to show that coiteration of mice up to some ordinal $\theta$ yields clubs below $\theta$ of critical points moved in the same way on both sides of the comparison.

Lemma 3. Let $\mathfrak{A} \models \mathrm{KP}$, with $\mathfrak{A}$ transitive, and let $M, N \in \mathfrak{A}$ be non-overlapping premice. Let $\theta=\mathrm{On}^{\mathfrak{A}}$. Suppose $M, N$ are coiterable for $\theta$ many steps (meaning that the models are wellfounded on both sides for this length of coiteration). Let $M_{\theta}, N_{\theta}$ be the direct limit models. Suppose $\theta \in \operatorname{WFP}\left(M_{\theta}\right) \cap \operatorname{WFP}\left(N_{\theta}\right)$. Then there is $C \subseteq \theta$, closed and unbounded in $\theta, C \in \Delta_{1}^{\mathfrak{A}}(M, N)$, with

$$
i<j \in C \longrightarrow \pi_{i, j}^{M}\left(\kappa_{i}\right)=\kappa_{j}=j=\pi_{i, j}^{N}\left(\kappa_{i}\right)
$$

where $\kappa_{i}$ is the critical point of the embeddings $\pi_{i, i+1}^{M}$ and $\pi_{i, i+1}^{N}$.

Proof: Although we have non-overlapping extenders here, this is just as for coherent sequences of measures, which is proven in [9]. The reader may also simplify the proof of the following Lemma.

Q.E.D.

Lemma 4. Let $\mathfrak{A}=\Sigma_{2}-\mathrm{KP}$, with $\mathfrak{A}$ transitive, and let $M, N \in \mathfrak{A}$ be type-0 premice. Let $\theta=\mathrm{On}^{\mathfrak{A}}$. Suppose $M, N$ are coiterable for $\theta$ many steps (again meaning that the models are wellfounded on both sides for this length of coiteration). Suppose $b, c$ are the cofinal branches on the $M$ (respectively $N$ ) side of the coiteration, and let $M_{b}, N_{c}$ be the direct limit models. Suppose $\theta \in \operatorname{WFP}\left(M_{b}\right) \cap \operatorname{WFP}\left(N_{c}\right)$. Then there is $C \subseteq \theta \cap b \cap c$, closed and unbounded in $\theta, C \in \Delta_{2}^{\mathfrak{A}}(M, N)$, with the equations of (1) above holding (and the same definitions of the $\kappa_{i}$ ).

Proof: We adapt the argument of [9]. Let $\delta \in b$ be sufficiently large so that for some $\alpha \in M_{\delta}, \pi_{\delta, \theta}^{M}(\alpha)=\theta$. Then, for $\gamma \in b \backslash \delta, \pi_{\delta, \gamma}^{M}(\alpha)={ }_{\mathrm{df}} \alpha_{\gamma} \geq \kappa_{\gamma}$. We wish to find $C_{0} \subseteq \theta \cap b \backslash \delta$, cub, for which $\alpha_{\gamma}=\kappa_{\gamma}=\gamma$. Let $\eta>\delta$ be chosen with $\eta \in b$. Suppose $\xi<\alpha_{\eta}$. By definition of $\alpha$, and thus $\alpha_{\eta}, \pi_{\eta, \theta}^{M}(\xi)<\theta$, and hence there is some $\nu>\eta$, $\nu \in b$, with $\pi_{\eta, \nu}^{M}(\xi)<\kappa_{\nu}$. By $\Sigma_{2}$-admissibility, we can thus define a function $f$, into $b$, so that if we have $f(\eta)=\nu$, then (a) $\nu \in b$; (b) for all $\xi<\alpha_{\eta} \pi_{\eta, \nu}^{M}(\xi)<\kappa_{\nu}$. (We need $\Sigma_{2}$-admissibility because $b, c \in \Pi_{1}(\mathfrak{A})$ not $\Delta_{1}(\mathfrak{A})$.) Thus $f$ is total, and $\Sigma_{2}(\mathfrak{A})$. Hence there is a $\Delta_{2}(\mathfrak{A})$ cub set $C_{0} \subseteq b \cap c$ so that $\gamma \in C_{0} \longrightarrow f^{\text {" }} \gamma \subseteq \gamma$. For such $\gamma \in C_{0}$ we have $\alpha_{\gamma}=\sup _{\eta \in b \cap \gamma} \kappa_{\eta}$ : if $\mu<\alpha_{\gamma}$ then $\mu=\pi_{\eta, \gamma}^{M}\left(\mu^{\prime}\right)$ for some $\eta<\gamma$ and $\mu^{\prime}<\alpha_{\eta}$; by the closure of $\gamma$ under $f$, we have that for some $\rho<\gamma \pi_{\eta, \rho}^{M}\left(\mu^{\prime}\right)<\kappa_{\rho}$. As $\operatorname{crit}\left(\pi_{\rho, \gamma}^{M}\right) \geq \kappa_{\rho}$ we have that $\mu<\kappa_{\gamma}$ as required.

Then we further refine $C_{0}$ to a cub $C \subseteq b \cap c$ by reasoning similarly on the $N$ side of the coiteration.

Q.E.D.

Straightforwardly generalising:

LEMMA 5. The above Lemma holds for type-1 premice providing $\mathfrak{A}=\Sigma_{3}-\mathrm{KP}$, and in the conclusion, $C$ is then $\Delta_{3}^{\mathfrak{A}}(M, N)$.

Lemma 6. Let $M, N$ be countable type-0 premice. Let $z \subseteq \omega$ code $M, N$. Then the comparison iteration with trees $\mathcal{T}, \mathcal{U}$ on $M, N$ respectively has length $<\zeta(2, z)$. 
Proof: Suppose otherwise, set $\zeta=\zeta(2, z)$ and let $\mathcal{T}, \mathcal{U}$ be the trees defined inside $\mathfrak{A}=L_{\zeta}[z]$, with lengths on both sides at least $\zeta$.

(1) The comparison can be continued without termination until $\Sigma(2, z)$.

Proof: Otherwise $L_{\Sigma}[z] \models=\exists \gamma\left(M_{\gamma}, N_{\gamma}\right.$ are terminal models in a successful comparison, or, $\gamma=\gamma^{\prime}+1$, and one of $M_{\gamma}, N_{\gamma}$ is illfounded; or $\operatorname{Lim}(\gamma)$, and if $e, f$ are the cofinal branches up to $\gamma$, then one of $M_{e}, N_{f}$ is illfounded)". However any of these alternatives would reflect down to $L_{\zeta}[z]$.

Q.E.D.(1)

Suppose then that the comparison trees $\mathcal{T}^{\prime}, \mathcal{U}^{\prime}$ are the result of continuing the comparison to stage $\Sigma$.

(2) Let $b, c$, be the respective cofinal branches to $\zeta$ of the $M, N$ sides respectively. Similarly let $b^{\prime}, c^{\prime}$ be the cofinal branches of $\mathcal{T}^{\prime}, \mathcal{U}^{\prime}$. Then $b^{\prime} \cap \zeta=b, c^{\prime} \cap \zeta=c$. Hence $\zeta \in b^{\prime} \cap c^{\prime}$.

Proof: By our assumption on $\Sigma_{2}$-elementarity, if $b, c$ are the $\Pi_{1}\left(L_{\zeta}[z]\right)$ cofinal branches through $\mathcal{T}, \mathcal{U}$, then they will be initial segments of $b^{\prime}, c^{\prime}$.

Q.E.D.(2)

Let $C \subseteq b \cap c$ come from Lemma 4. By our properties of $C$ :

(3) $\xi \in b \cap c \cap C \longrightarrow \operatorname{crit}\left(\pi_{\xi, \zeta}^{M}\right)=\operatorname{crit}\left(\pi_{\xi, \zeta}^{N}\right)=\xi$.

We may also define $C^{\prime}$ in a $\Delta_{2}$ fashion over $L_{\Sigma}[z]$ from $M, N$ just as $C$ was defined over $L_{\zeta}[z]$ : although $L_{\Sigma}[z]$ is not admissible for $\Sigma_{2}$ formulae for all choices of parameters, it is so admissible for choices of parameters (here $M, N$ ) in $L_{\zeta}[z]$ itself, which is all we require here. Just as for the branches $b^{\prime}, c^{\prime}$ we have that $C^{\prime} \cap \zeta=C$, using the $\Sigma_{2}$ elementarity of $L_{\zeta}[z]$ in $L_{\Sigma}[z]$.

Consider the following definitions about any $\xi$ which is a limit point of $b \cap c \cap C$.

Let $\mu, \eta$ be such that the $b$ (respectively $c$ ) successors of $\xi$ are $\mu+1$, respectively $\eta+1$. Further let $\nu=\nu_{\mu}=\nu_{\eta}$ be the index of the least difference of the $E^{M_{\mu}}, E^{N_{\eta}}$ hierarchies, and let some $a \in[\nu]^{<\omega}$ be chosen least with some least $A \in \mathcal{P}\left([\xi]^{|a|}\right) \cap\left(E_{\nu, a}^{M_{\mu}} \Delta E_{\nu, a}^{N_{\eta}}\right)$. Then let $\delta=\delta(\xi) \in C \cap \xi$ be sufficiently large so that there exist $a(M), \nu(M), A(M)(a(N), \nu(N), A(N)) \in$ $M_{\delta}\left(\right.$ respectively $\left.N_{\delta}\right)$ with $\pi_{\delta, \xi}^{M}(\nu(M))=\nu=\pi_{\delta, \xi}^{N}(\nu(N))$ and $\pi_{\delta, \xi}^{M}(a(M))=$ $a=\pi_{\delta, \xi}^{N}(a(N)) \wedge \pi_{\delta, \xi}^{M}(A(M))=A=\pi_{\delta, \xi}^{N}(A(N))$.

Now, in the above take $\xi=\zeta$ and $\delta=\delta(\zeta)$. Hence the following holds:

$L_{\Sigma}[z]=$ " $\exists \lambda \in b^{\prime} \cap c^{\prime} \cap C^{\prime} \exists \mu^{\prime}, \eta^{\prime}\left[\mu^{\prime}+1, \eta^{\prime}+1\right.$ are the $T, U$ successors of $\lambda=\kappa_{\lambda}=\pi_{\delta, \lambda}^{M}(\delta)=\pi_{\delta, \lambda}^{N}(\delta) \wedge$ (letting $\nu=\nu_{\mu^{\prime}}=\nu_{\eta^{\prime}}$ and $a \in[\nu]^{<\omega}$ be least so that there is some $A$ with

$$
\pi_{\delta, \lambda}^{M}(A(M))=A=\pi_{\delta, \lambda}^{N}(A(N)) \in E_{\nu, a}^{M_{\mu^{\prime}}} \Delta E_{\nu, a}^{N_{\eta^{\prime}}}
$$

with $\operatorname{crit}\left(E_{\nu}^{M_{\mu^{\prime}}}\right)=\operatorname{crit}\left(E_{\nu}^{N_{\eta^{\prime}}}\right)=\lambda$, we have that $\pi_{\delta, \lambda}^{M}(a(M))=a=$ $\left.\left.\pi_{\delta, \lambda}^{N}(a(N))\right)\right] . "$

Namely, take $\lambda=\zeta$.

This is a $\Sigma_{2}$ statement (coming just from the outer quantifier " $\exists \lambda \in b^{\prime} \cap c^{\prime} \cap C^{\prime \prime}$ ") about $M, N, \delta, a(M), a(N), A(M), A(N)$ and $b^{\prime}, c^{\prime}, C^{\prime}$, and hence goes down to $L_{\zeta}[z]$ about 
those same objects and the branches to $\zeta, b, c$ and $C$. Let $\lambda, \mu^{\prime}, \eta^{\prime}, \nu, a, A$ instantiate this in $L_{\zeta}[z]$. Then as (i) $\delta \in C$, (ii) $\lambda \in b \cap c \cap C$, and (iii) $\lambda=\kappa_{\lambda}=\pi_{\delta, \lambda}^{M}(\delta)=\pi_{\delta, \lambda}^{N}(\delta)$ we have then $\pi_{\lambda, \zeta}^{M}(\lambda)=\pi_{\lambda, \zeta}^{N}(\lambda)$. Hence $A=\pi_{\lambda, \zeta}^{M}\left(A \cap[\lambda]^{|a|}\right)=\pi_{\lambda, \zeta}^{N}\left(A \cap[\lambda]^{|a|}\right)$, whilst at the same time $a \in \pi_{\lambda, \zeta}^{M}\left(A \cap[\lambda]^{|a|}\right) \longleftrightarrow a \notin \pi_{\lambda, \zeta}^{N}\left(A \cap[\lambda]^{|a|}\right)$ which is absurd.

Q.E.D.

Lemma 7. Let $M, N$ be countable type-1 premice. Suppose $z$ codes $M, N$. Then the comparison iteration with trees $\mathcal{T}, \mathcal{U}$ on $M, N$ respectively have length $<\zeta(3, z)$.

Proof: The argument is identical to the last Lemma, where instead we appeal to Lemma 5 , and show that $L_{\zeta}[z]$ is a $\Sigma_{3}$-KP model, and we substitute $\Sigma_{3}$-reflection for $\Sigma_{2}$.

Q.E.D.

We now finish off the theorems by running Steel's argument directly. The reader familiar with this will find no novelty in what follows.

Proof of Theorem 6. Suppose $V=L[E]$ and there are only type-0 premice. We show that the $<_{L[E]}$ wellorder of the reals is $\partial \Gamma_{2}$. For $x, y \in \omega_{\omega}$ players $I$ and $I I$ play a game $G_{x, y}$ producing integers $n_{0}, n_{1}, \ldots$ alternately, and constructing reals $u=$ $\left(n_{0}, n_{2}, \ldots, n_{2 n}, \ldots\right), v=\left(n_{1}, n_{3}, \ldots, n_{2 n+1}, \ldots\right)$, and thus a $z=u \oplus v$ in the usual way.

Rules: I's real $u$ must code a premouse $M=M_{0} \models$ “ $x<_{M} y$ " in the standard order of construction of the $M=L\left[E^{M}\right]$ hierarchy. If he fails at this he loses. If he succeeds, then II's real $v$ must similarly code a premouse $N=N_{0} \models$ " $y \leq_{N} x$ " (or else she will lose).

If both players have passed these tests, then we form the coiteration of $M, N$ to $\zeta=\zeta(2, z)$. Player $I$ wins $G_{x, y}$ iff $\exists \xi \leq \zeta\left(M_{\xi}\right.$ is wellfounded, but $N_{\xi}$ is not).

This is then a $\Gamma_{2}$ relation of $x, y, u, v$, the winning conditions for $I$ being expressible in a $\Sigma_{1}$ way over $L_{\zeta}[z]$ (or equivalently over $L_{\Sigma(2, z)}$ ). It is now easy to see that if $x<_{L[E]} y$ then $I$ has a winning strategy which is to play out $u$, a code for the least countable level $M=L_{\alpha}[E]$ witnessing this. He can ignore $I I$ 's efforts. On the one hand we can never have a properly terminating coiteration (meaning with both terminating models wellfounded) of the two models $M, N$ as they disagree fundamentally about the ordering of their reals. By Lemma 6 however, the comparison must terminate before $\zeta$. However, it can only terminate through one of the models being illfounded. This has to be $I I$ 's model. Thus $I$ wins the game. If $y \leq_{L[E]} x$ the argument is symmetrical with $I I$ winning.

Q.E.D.

The proof of Theorem 7 is entirely similar $m . m$. If $\operatorname{Det}\left(\Pi_{1}^{1}\right.$-IND) holds in $V$ then $a$ fortiori we have $\operatorname{Det}\left(\Pi_{1}^{1}\right)$. This yields, by work of Martin, and a final theorem of Harrington [5] that for any real $x x^{\#}$ exists. From this we know by [11] that, assuming there is no inner model with a strong cardinal, that the core model $K$ is $\Sigma_{3}^{1}$-absolute. Since $\operatorname{Det}\left(\Pi_{1}^{1}\right.$-IND) can be written as a $\Sigma_{3}^{1}$ sentence it must be true in $K$ which is a model of the form $L[E]$. However this contradicts Theorem 5. hence there is such an inner model and Theorem A holds. Theorem B is similar, but we do need a little more than $\forall x x^{\#}$ currently to establish this (we believe it is still an open problem as to whether this extra is needed); Schindler has shown (unpublished) that Steel's result on the $\Sigma_{3}^{1}$ 
correctness of $K$ (which required an assumption of two measurable cardinals see [10]) can be effected using an assumption of only that $\forall x\left(\left(x^{\dagger}\right)^{\#}\right.$ exists). This is equivalent by an unpublished theorem of Martin to $\operatorname{Det}\left(\omega^{2}+1-\Sigma_{1}^{\mathbf{1}}\right)$. This is the pointclass formed from the $\omega^{2}+1$-st level of the Hausdorff difference hierarchy on analytic sets. This level of determinacy is weaker than both that of $\Pi_{1}^{1}$-IND and AQI. Hence the same argument proves Theorem B.

It is possible to envisage other kinds of quasi-inductive process generalising the operators $\Gamma$ mentioned above. These operators use limit rules that are $\Sigma_{n}$ (rather than the $\Sigma_{2}$ used above for AQI). The "stability sets" $\Gamma^{\infty}(z)$ derived from these processes stabilize then at, or by, the ordinals $\zeta(n, z)$ above. Examples of such operators can be found in [4]. Let us call $A Q I_{n}$ the abstract pointclass where such a $\Sigma_{n}$ rule is used and $\mathbf{A Q I} \mathbf{I}_{n}$ the boldface counterpart. (We are deliberately being somewhat vague here as we do not wish to go into the complexities of the definitions from [4]. Suffice it to say we are imagining some form of $\Sigma_{n}$-limit rule modelled along the lines of the " $\Sigma_{n}$-rule" defined there.) Using these, abstract versions of the above for mice requiring iteration trees with $\Pi_{n}$ definable branches and $\Sigma_{n}$ reflection, are provable but what kind of mice or iteration trees are these?

There is then a Theorem $\mathrm{C}$ which runs as follows.

Theorem $\mathbf{C} \operatorname{Det}\left(\mathbf{A Q I} \mathbf{I}_{3}\right) \Rightarrow$ there exist type $\geq 2$ mice.

Clearly the above proofs generalise if one has a natural class of premice whose iteration trees have $\Pi_{n}$ definable cofinal branches, and which can be used in generalisations of the above proofs for $\Gamma_{n}$ for $n>3$.

On seeing the above arguments Ralf Schindler suggested to check the mice below $0^{n !}$ of [8] Def. 2.10. These are the " $n$-hand-grenades." We repeat Schindler's definition, but leave this matter open here.

Definition 8. Let $M$ be a premouse, and let $\mu<\kappa \leq O n^{M}$. We call $\mu<\kappa$-0strong in $M$ if $\mu$ is a measurable cardinal in $M$ as witnessed by $E^{M}$, i.e, there is an extender $E_{\omega \nu}^{M}$ with critical point $\mu$ and such that $\nu>\left(\mu^{+}\right)^{M}$.

For $n<\omega$ we call $\mu<\kappa$ - $(n+1)$ - strong in $M$ if $\mu$ is $<\kappa$-strong in $M$ as witnessed by $E^{M}$ and there are arbitrary large $\gamma<\mu$ such that $\gamma$ is $<\mu$-n-strong in $M$.

DEFINITION 9. Let $M$ be a premouse, and let $n \geq 1$. $M$ is said to be below $0^{n !}$ if there is no $\kappa$ which is the critical point of an extender $E_{\nu}^{M} \neq \varnothing$, with the property that $\{\mu<\kappa \mid \mu$ is $<\kappa$-n-strong in $M\}$ is unbounded in $\kappa$.

Then mice below $0^{!}=0^{1 !}$ correspond to type- 0 in the Feng-Jensen definition above, and comparison of such premice results in almost linear iteration trees.

Question 1: Is there a characterisation of premice of type- $n$ as having branches of complexity $\Pi_{n+1}$ through their iteration trees? Conversely, are the premice whose branches are at this level of complexity those below $0^{n+1 !}$ ?

Conjecture 1: Let $n>3$. Then $V=L[E] \wedge \operatorname{Det}\left(A Q I_{n}\right)$ implies the existence of $0^{n !}$. 
(The cases $n=2,3$ come from the correspondence between the Feng-Jensen mice and the hand-grenades, and Theorems 6,7 above.)

Schindler mentions another mouse, let us call it for the nonce, of "stationary strong type": this is a premouse $M$ with a top extender $F$ with $\operatorname{crit}(F)=\kappa$ where

$$
M \models “\left\{\mu<\kappa \mid \mu \text { is }<\kappa \text {-strong as witnessed by } E^{M}\right\} \text { is stationary.” }
$$

It is then easy to see that for every $n M$ is beyond $0^{n !}$. Suppose the answer to the last part of Question 1 is affirmative; let $Z_{2}$ be the theory of analysis, then we should have a proof of the following conjecture:

Conjecture 2: $Z_{2}$ cannot prove that any two premice of stationary strong type are comparable.

Even with the above analysis we do not know the answer to:

Question 2: Are these estimates of the amount of determinacy available in the corresponding inner models $L[E]$ given by the above theorems anywhere near the best possible?

We should finally caution that all of the above discussion only scratches the surface of the levels of determinacy obtainable in the Steel core model $K$ ([10]) below a Woodin cardinal.

REFERENCES

[1] P. ACZEL and W. RICHTER, Inductive definitions and reflecting properties of admissible ordinals, Generalised recursion theory (Proc. Symp., Oslo, 1972), Studies in Logic and the Foundation of Mathematics,, vol. 79, North-Holland, Amsterdam, 1974, pp. 301-381.

[2] J.P. BURGESS, The truth is never simple, this JOURNAL, vol. 51 (1986), no. 3, pp. 663-681.

[3] Q. FEng and R.B. JENSEN, Supercomplete extenders and type 1 mice, Annals of Pure and Applied Logic, vol. 128 (2004), no. 1-3, pp. 1-73.

[4] S. D. FRIEDMAN and P. D. WeLCh, Hypermachines, this JouRnal, (to appear).

[5] L. HARrington, Analytic determinacy and 0\#, this Journal, vol. 43 (1978), no. 4, pp. 684-693.

[6] A. S. KeChrIS, Forcing in analysis, Higher set theory (G. Müller and D. Scott, editors), Lecture Notes in Mathematics, vol. 669, Springer, pp. 272-302.

[7] Y. N. Moschovakis, Descriptive set theory, Studies in Logic series, North-Holland, Amsterdam, 1980.

[8] R-D. SCHINDLER, The core model for almost linear iterations, Annals of Pure and Applied Logic, (2002), pp. 205-272.

[9] J. R. STEeL, Determinacy in the Mitchell models, Annals of Mathematical Logic, vol. 22 (1980), pp. $109-125$. 1996.

[10] - The core model iterability problem, Lecture Notes in Mathematical Logic, vol. 8, Springer,

[11] J. R. STEEL and P. D. WELCH, $\Sigma_{3}^{1}$-absoluteness and the second uniform indiscernible, Israel Journal of Mathematics, vol. 104 (1998), pp. 157-190.

[12] P. D. WELCH, The length of infinite time Turing machine computations, Bulletin of the London Mathematical Society, vol. 32 (2000), pp. 129-136.

[13] — Post's and other problems in higher type supertasks, Classical and new paradigms of computation and their complexity hierarchies, papers of the conference foundations of the formal sciences iii (B. Löwe, B. Piwinger, and T. Räsch, editors), Trends in logic, vol. 23, Kluwer, Oct 2004, pp. 223-237. 
SCHOOL OF MATHEMATICS,

UNIVERSITY OF BRISTOL,

BRISTOL,

BS8 1TW,

ENGLAND

E-mail: p.welch@bristol.ac.uk 